\title{
IDADE MATERNA X SÍNDROME DE DOWN EM QUATRO \\ MUNICÍPIOS DO CENTRO-OESTE MINEIRO
}

\author{
Daniela da Cruz Teodoro \\ Bióloga - e-mail:danibiologa88@yahoo.com.br \\ Lília Rosário Ribeiro \\ Professora Adjunta do UNIFOR-MG \\ e-mail: lilia@uniformg.edu.br \\ Aladir Horácio dos Santos \\ Professor Adjunto do UNIFOR-MG \\ e-mail: aladirhs@oi.com.br
}

\begin{abstract}
RESUMO
A Síndrome de Down é uma das alterações cromossômicas mais comuns em humanos e pode surgir como consequência de uma trissomia livre do cromossomo 21, translocação 14/21 ou mosaicismo. Este trabalho objetivou a realização de um estudo sobre as idades maternas dos portadores da Síndrome de Down matriculados em instituições especializadas em atendimento a portadores de necessidades especiais de quatro cidades da região Centro-Oeste de Minas Gerais no ano de 2008. O trabalho mostrou uma correlação de 0,5927 entre a idade materna e o número de portadores da Síndrome de Down. Das comparações feitas com o grupo que apresentou a Síndrome de Down e o grupo controle, foi obtida uma média de idade materna de 34,4 anos para os portadores e uma média igual a 28,0 anos para o grupo controle, o que confirma a influência da idade materna avançada no surgimento da Síndrome de Down.
\end{abstract}

Palavras-chave: Idade Materna. Síndrome de Down. Trissomia.

\section{MATERNAL AGE X DOWN'S SYNDROME IN FOUR CITIES OF "MINAS GERAIS" MIDWEST}

\begin{abstract}
Down's Syndrome is one of the most common chromosomal changes in humans and may arise from free trisomy of chromosome 21, translocation $14 / 21$ or mosaicism. This work aimed at achieving a study about Down's Syndrome carriers' maternal ages enrolled in institutions specialized in caring for special needs carrier's in four towns in central-west region of Minas Gerais in the year of 2008. The study showed a correlation of 0, 5927 between maternal age and a number of Down's Syndrome carriers. Comparisons made between the group which had Down's Syndrome and the control group obtained an average maternal age of 34, 4 years old for the carriers and an average equal to 28,0 years old for the control group, which confirms the influence of advanced maternal age at onset of Down's Syndrome.
\end{abstract}

Keywords: Maternal Age. Down's Syndrome. Trisomy.

\section{INTRODUÇÃO}

A Síndrome de Down foi descrita, pela primeira vez, por Langdon Down, em 1866, como sendo 
uma doença congênita multissistêmica. Ela é, geralmente, caracterizada pela combinação de retardo mental e várias malformações físicas, como prega epicântica larga, pescoço largo, curto, anomalias cardíacas, intestinais, alterações visuais, problemas nas articulações e músculos, olhos com fissura palpebral oblíqua, entre outros. (BERTHOLD et al, 2004).

A Síndrome de Down apresenta uma frequência de 0,15\% dos nascidos vivos. Os indivíduos portadores desta Síndrome têm uma cópia extra do cromossomo 21, causada por uma não-disjunção em um dos genitores. Alguns casos podem surgir por translocações 14/21 ou mosaicismo. (GRIFFITHS et al, 2006).

De acordo com Gusmão, Tavares e Moreira (2003), a idade reprodutiva está relacionada à origem de trissomias e a idade materna avançada, acima de 35 anos, é uma variável fortemente associada à Síndrome de Down.

O aumento da taxa da Síndrome de Down em progênies de mães cuja idade ultrapassa 35 anos é devido à soma de, no mínimo, dois componentes, um indepen- dente da idade materna, outro dependente. Erros na formação de quiasmas, pareamento de cromossomos meióticos e decréscimo da seleção dos óvulos em mulheres com idade avançada estão relacionados à Síndrome de Down. Outra possível causa seria a predisposição genética para a não-disjunção. Pesquisas apontam que determinadas famílias podem ter essa característica. (GUSMÃO; TAVARES; MOREIRA, 2003). Mães com idades entre 40 e 44 anos são responsáveis por somente $2 \%$ do total de nascimentos. Todavia, desse total, 40\% das crianças nascem com a Síndrome de Down. (CASTILLA et al,1995).

Diversos relatos sobre a influência da idade materna avançada e a incidência da Síndrome de Down são existentes na literatura (AYMÉ; LIPPMAN-HAND, 1982; BEIGUELMAN; KRIEGER; SILVA, 1996; BERTHOLD et al, 2004; GUSMÃO; TAVARES; MOREIRA, 2003; LAMSON; HOOK, 1981; NAKADONARI; SOARES, 2006), entretanto, dados do interior de Minas Gerais não são disponí- veis.

Este trabalho teve como objetivo realizar um estudo sobre a Síndrome de Down e sua correlação com a idade materna avançada em portadores de quatro municípios da região Centro-Oeste de Minas Gerais: Lagoa da Prata, Formiga, Arcos e Piumhi.

\section{MATERIAIS E MÉTODOS}

Este trabalho foi avaliado pelo Comitê de Ética em Pesquisa Envolvendo Humanos do UNIFOR-MG e obedeceu a todos os preceitos éticos e legais. A amostra dos portadores da Síndrome de Down analisados neste trabalho foi constituída de 27 portadores da cidade de Formiga, 12 portadores da cidade de Arcos, 10 portadores da cidade de Piumhi e 17 portadores da cidade de Lagoa da Prata, matriculados em instituições especializadas em atendimento a portadores de necessidades especiais dos respectivos municípios, no ano de 2008, totalizando 66 portadores. Como grupo controle, tomou-se uma amostra 
casual de 200 indivíduos pertencentes a uma escola pública do município de Formiga - MG.

As idades maternas dos portadores foram comparadas estatisticamente com as idades maternas do grupo controle, por meio do teste t de Student, e as diferenças analisadas através do teste do qui quadrado $\left(c_{2}\right)$, de modo a verificar se existe uma correlação direta entre a incidência da Síndrome de Down e a idade materna avançada. O nível de significância adotado para as comparações foi de 5\%.

\section{RESULTADOS E DISCUSSÕES}

A TAB.1 mostra a distribuição das crianças com Síndrome de Down da cidade de Formiga-MG.

Tabela 1 - Distribuição da Síndrome de Down de acordo com a idade materna em portadores da cidade de Formiga-MG

\begin{tabular}{l|l}
\hline Idade materna (anos) & Número de portadores da Síndrome de Down \\
$15-20$ & 1 \\
$20-25$ & 1 \\
$25-30$ & 4 \\
$30-35$ & 5 \\
$35-40$ & 8 \\
$40-45$ & 6 \\
$45-50$ & 2 \\
\hline
\end{tabular}

Os dados do GRAF. 1 mostram que o maior número de portadores com Síndrome de Down é coincidente com a idade materna entre 35 e 40 anos, concordando com os dados de Gusmão, Tavares e Moreira (2003).

Gráfico 1 - Distribuição da Síndrome de Down de acordo com a idade materna em portadores da cidade de Formiga-MG

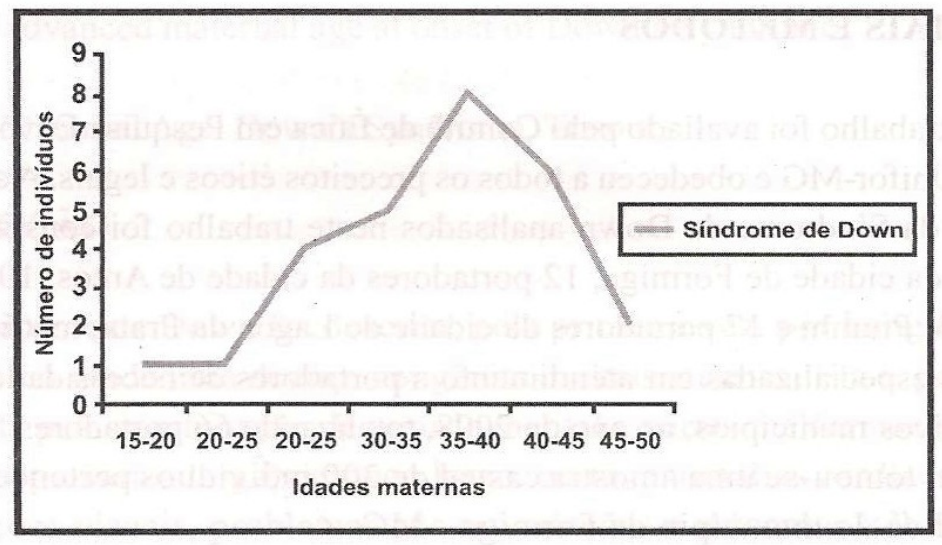

A distribuição de portadores e as referidas idades maternas da cidade de Arcos é mostrada na TAB.2. Verifica-se que houve maior número de portadores quando as idades maternas encontraram-se na faixa de 35 a 40 anos. Concordando com Moreira e Gusmão (2002), que explicam que a possibilidade da ocorrência da Síndrome de Down aumenta com a idade materna, devido ao processo de formação precoce das células germinativas femininas, que, ao longo dos anos, vão sofrendo 
alterações.

Tabela 2 - Distribuição da Síndrome de Down de acordo com a idade materna em portadores da cidade de Arcos-MG

\begin{tabular}{l|l}
\hline Idade materna (anos) & Número de portadores da Síndrome de Down \\
$10-15$ & 1 \\
$15-20$ & 0 \\
$20-25$ & 2 \\
$25-30$ & 1 \\
$30-35$ & 1 \\
$35-40$ & 6 \\
$40-45$ & 1 \\
\hline
\end{tabular}

O GRAF. 2 mostra a variação da idade materna e o pico de incidência da Síndrome de Down nos portadores da cidade de Arcos-MG.

Gráfico 2 - Distribuição da Síndrome de Down de acordo com a idade materna em portadores da cidade de Arcos-MG

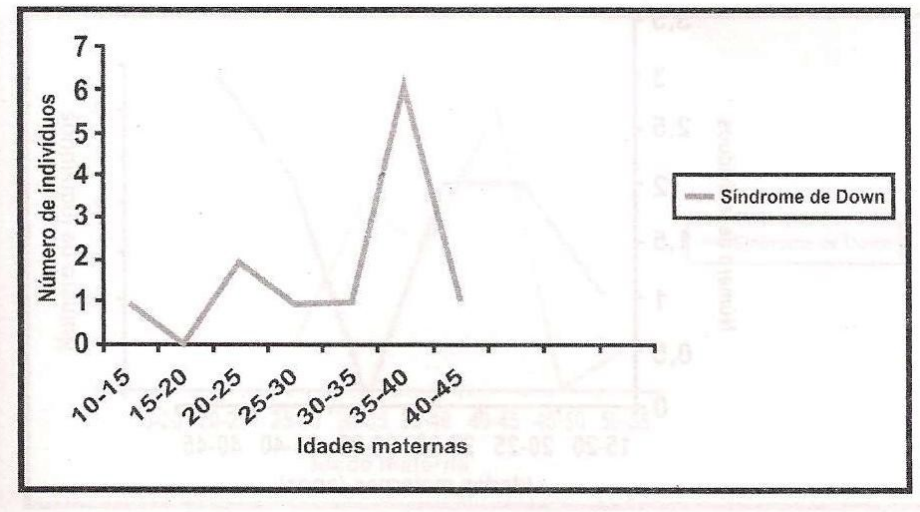

Segundo Siqueira (2006), a idade materna reflete numa capacidade de, cada vez mais, mães idosas abortarem os zigotos anormais. Assim, o aumento da idade materna estará relacionado com a redução da recombinação meiótica, de que resultará uma separação prematura das cromátides e a nãodisjunção cromossômica durante a primeira divisão da meiose.

A TAB. 3 apresenta a distribuição de portadores da Síndrome de Down da cidade de Piumhi e as respectivas idades maternas. O GRAF. 3 mostra que o pico de incidência da Síndrome de Down coincide com o intervalo de idade materna entre 40 e 45 anos.

Tabela 3 - Distribuição da Síndrome de Down de acordo com a idade materna em portadores da cidade de Piumhi-MG

\begin{tabular}{l|l}
\hline Idade materna (anos) & Número de portadores da Síndrome de Down \\
$15-20$ & 1 \\
$20-25$ & 2 \\
$25-30$ & 2 \\
$30-35$ & 0 \\
$35-40$ & 2 \\
$40-45$ & 3 \\
\hline
\end{tabular}


Gráfico 3 - Distribuição da Síndrome de Down de acordo com a idade materna em portadores da cidade de Piumhi-MG

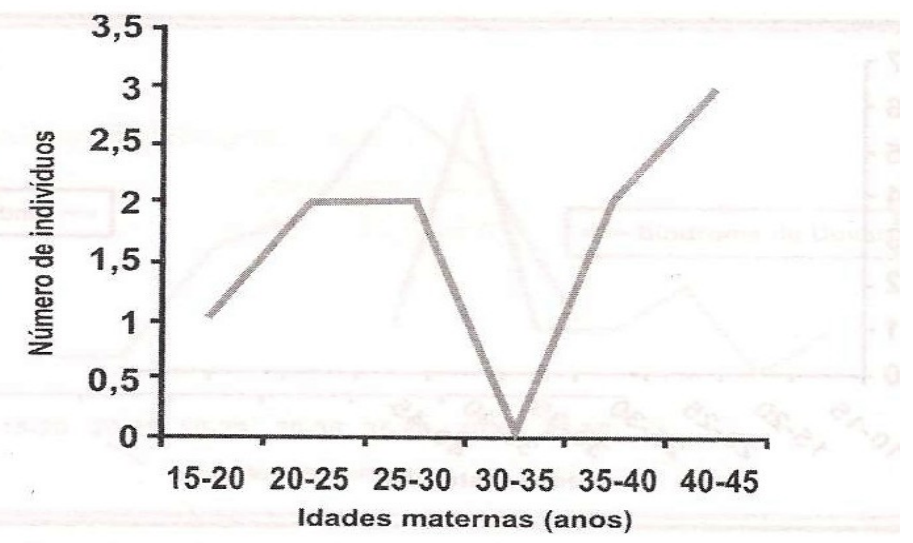

É possível observar nesta distribuição que o número de portadores foi mais elevado quando a idade materna ultrapassou os 35 anos. Alguns estudos como os de Gusmão, Tavares e Moreira (2003) apontam que $40 \%$ dos nascidos com esse tipo de Síndrome têm mães com idade entre 40 e 44 anos.

A TAB. 4 apresenta o número de portadores de Síndrome de Down da cidade de Lagoa da Prata e as respectivas idades maternas.

Tabela 4 - Distribuição da Síndrome de Down de acordo com a idade materna em portadores da cidade de Lagoa da Prata-MG

\begin{tabular}{l|l}
\hline \multicolumn{1}{c|}{ Idade materna (anos) } & Número de portadores da Síndrome de Down \\
$15-20$ & 1 \\
$20-25$ & 1 \\
$25-30$ & 1 \\
$30-35$ & 4 \\
$35-40$ & 3 \\
$40-45$ & 6 \\
$45-50$ & 0 \\
$50-55$ & 1 \\
\hline
\end{tabular}

A incidência da Síndrome de Down na cidade de Lagoa da Prata foi maior quando a idade materna situou-se no intervalo entre 40 e 45 anos. (GRAF. 4).

Gráfico 4 - Distribuição da Síndrome de Down de acordo com a idade materna em portadores da cidade de Lagoa da Prata-MG

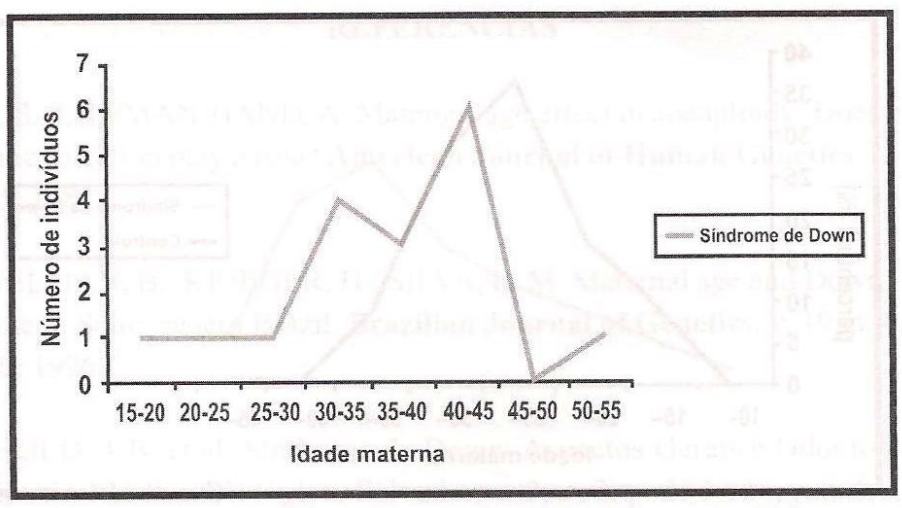


A distribuição dos portadores da Síndrome de Down das quatro cidades da região Centro-Oeste em estudo e o grupo controle, em função da idade materna, são mostrados na TAB 5. O GRAF. 5 mostra essa distribuição. Observa-se que a idade das mães com filhos portadores da Síndrome de Down é significativamente maior do que a de mães cujos filhos não apresentam a Síndrome, conforme demonstrado pela análise não-paramétrica e pela distribuição dos dados da TAB.5 $\left(c_{2}=52,035\right.$; GL= 7; $\mathrm{P}=0,0001)$.

Tabela 5 - Distribuição geral do número de portadores da Síndrome de Down e do grupo controle de acordo com a idade materna

\begin{tabular}{l|l|l}
\hline Idade materna (anos) & Síndrome de Down & Grupo controle \\
$10-15$ & 2 & 0 \\
$15-20$ & 5 & 9 \\
$20-25$ & 9 & 17 \\
$25-30$ & 12 & 36 \\
$30-35$ & 17 & 28 \\
$35-40$ & 27 & 9 \\
$40-45$ & 22 & 1 \\
$45-50$ & 6 & 1 \\
Média & 34,4 & 28,0 \\
Desvio padrão & 8,3 & 5,7 \\
\hline $\mathrm{c}^{2}=52,035 ; \mathrm{GL}=7 ; \mathrm{P}=0,0001$
\end{tabular}

Gráfico 5 - Distribuição percentual dos portadores da Síndrome de Down e do grupo controle de acordo com a idade materna

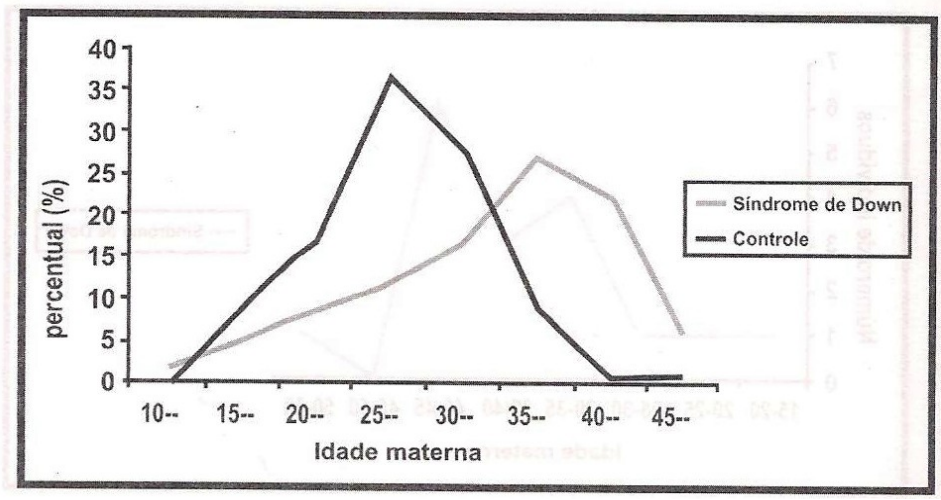

Os resultados obtidos nesse trabalho mostram que as idades maternas dos portadores é significativamente superior $(\mathrm{t}=8,98 ; \mathrm{p}=0,0001)$ às do grupo controle. A maior distribuição percentual de portadores da Síndrome de Down é revelada no pico de idade materna entre 35 a 45 anos. A média das idades maternas dos portadores foi de 34,4 anos, com desvio padrão de 8,3. Já a média do grupo controle foi de 28,0 anos, com desvio padrão de 5,7.

Por meio de uma correlação feita entre o total de portadores da Síndrome de Down e as idades maternas dos mesmos, existe um índice de valor = 0,5927 entre a idade materna e a Síndrome. 


\section{CONCLUSÃO}

Os resultados obtidos neste trabalho revelam que a incidência da Síndrome de Down nas quatro cidades (Formiga, Arcos, Piumhi e Lagoa da Prata) do Centro- Oeste mineiro foi aumentada quando a idade materna esteve entre 35 e 45 anos, reafirmando assim, os estudos apresentados até o momento, que indicam uma correlação direta entre a incidência da Síndrome de Down e a idade materna avançada.

\section{REFERÊNCIAS}

AYMÉ, S.; LIPPMAN-HAND, A. Maternal-age effect in aneuploidy: does altered embrionic selection play a role? American journal of human genetics, USA, v. 34, p. 558-565, 1982.

BEIGUELMAN, B.; KRIEGER, H.; SILVA, L. M. Maternal age and Down syndrome in Southeastern Brazil. Brazilian journal of genetics, São Paulo, v. 19, n. 4, p. 637-640, 1996.

BERTHOLD, T.B. et al. Síndrome de Down: aspectos gerais e odontológicos. Ciência médica biológica, Salvador, v. 3, n. 2, p. 252-260, jul./dez. 2004.

CASTILlA et al. Atlas de lãs malformações congênitas em Sudamérica. Rio de Janeiro: Fiocruz, 1995.

GUSMAO, F. A. F.; TAVARES, E. J. M.; MOREIRA, L. M. A. Idade materna e síndrome de Down no nordeste do Brasil. Cadernos saúde pública, Rio de Janeiro, v. 19, n. 4, 2003. Disponível em: $<$ http://www.scielosp.org/scielo.php?script=sci_arttext\&pid= S010211X2003000400020\&lng=pt\& nrm=iso $>$. Acesso em: 04 maio 2008.

GRIFFTHS, A. J. F. et al. Introdução à genética. 8. ed. Rio de Janeiro: Guanabara Koogan, 2006.

LAMSON, S. H.; HOOK, E. B. Comparison of mathematical models for the maternal age dependence of Down's syndrome rates. Human genetics, USA, v. 59, n. 3, Dec. 1981.

MOREIRA, L. M. A.; GUSMAO, F. A. F. Aspectos genéticos e sociais da sexualidade em pessoas com síndrome de Down. Revista brasileira de psiquiatria, São Paulo, v. 24, n. 2, 2002. Disponível em: $<$ http://www.scielo.br/scielo.php?script=sci_arttext\&pid=S15164462002000200011\&lng= pt\&nrm $=$ iso $>$. Acesso em: 15 jun. 2008.

NAKADONARI E. K.; SOARES A. A. Síndrome de Down: considerações gerais sobre a influência da idade materna avançada. Arquivos do MUDI, Maringá, v. 10, n. 2, p. 5-9, 2006.

SIQUEIRA, V. Síndrome de Down: translocação robertsoniana. Saúde \& ambiente em revista, Duque de Caxias, v.1, n.1, p.23-29, jan./jun. 2006. 